J. Clin. Chem. Clin. Biochem.

Vol. 28, 1990, pp. $913-918$

(C) 1990 Walter de Gruyter \& Co. Berlin $\cdot$ New York

\title{
An Extraction/Enzymatic Procedure for Serum Cholesterol Measurement: Evaluation of Performance Characteristics
}

\author{
By C. Franzini ${ }^{1}$ and Paola Luraschi ${ }^{2}$ \\ 1 Laboratorio Analisi, Ospedale di Rho, Rho (Milano), Italy \\ ${ }^{2}$ Laboratorio Analisi, Istituto Scientifico S. Raffaele, Milano, Italy
}

(Received June 18/August 6, 1990)

\begin{abstract}
Summary: The performance characteristics of an extraction/enzymatic procedure for serum cholesterol measurement were evaluated. The procedure is substantially derived from the accepted reference method as standardized by the Centers for Disease Control, substituting the enzymatic reaction for the LiebermannBurchard reaction. Imprecision (CV) was consistently $<1.5 \%$, and accuracy was comparable to that of the definitive isotope dilution mass spectrometry method and the accepted reference method. Direct comparison of the enzymatic with the Liebermann-Burchard reaction, using a set of 50 human sera, revealed about $-0.05 \mathrm{mmol} / \mathrm{l}$ constant bias of the former versus the latter, this being possibly due to higher specifity of the enzymatic reaction. As compared with the accepted reference method, the method described is characterized by higher practicability, the reagent being easier to prepare and to handle, and generating a more stable, chemically defined end-product.
\end{abstract}

\section{Introduction}

The correct approach for an effective control of accuracy in clinical chemistry has been discussed in general terms by Tietz, in 1979 (1). In the case of the measurement of serum cholesterol concentration, the implementation of a "Reference System" (2) has been made possible through the availability of a definitive method (3) based on isotope dilution-mass spectrometry (IDMS), and a reference method (AK-CDC) based on the Abell-Kendall procedure, including the Liebermann-Burchard reaction, as rigorously defined by the Centers for Disease Control (CDC) (4).

In the process of transferring accuracy from the higher-class analytical level to the routine, every-day every-system level, a key role is played by the reference method. This should be used for the direct assessment of the accuracy of each routine method, in measuring cholesterol concentration in fresh patient sera (2). In this operation, the utilization of the definitive method is hindered by its low practicability. It follows that the reference method, whilst maintaining accuracy comparable to the definitive one, should be practicable enough to be performed widely.

Suggested alternatives to the reference (AK-CDC) method include gaschromatographic (5) and extraction/enzymatic $(6,7)$ techniques. In the latter, the cholesterol oxidase/peroxidase/chromogenic acceptor reaction (8) replaces the Liebermann-Burchard reaction in an AK-CDC-like analytical procedure. Advantages of the former over the latter reaction are two-fold: from the practical point of view, the enzymatic reagent is available in pre-packed form, is a less viscous and neutral solution, and it generates a more stable chromogen; from the theoretical point of view, the enzymatic reaction is more specific for cholesterol, and it gives rise to a chemically defined component.

In 1984 (6) we reported favourable results in our use of the extraction/enzymatic procedure, and suggested its use as a possible alternative for reference-class measurements. In this paper we report further results concerning the analytical reliability of the extraction/ enzymatic procedure. 


\section{Materials and Methods}

Analytical-grade chemicals (from Merck) were used throughout. Pure cholesterol for calibration (reportedly equivalent to NBS) and the enzymatic cholesterol reagent, available as a testkit (CHOD-PAP, High Performance), were from BoehringerMannheim; final concentrations in the reagent, reconstituted as recommended by the manifacturer, are (9):

TRIS- $\mathrm{HCl}$ buffer, $\mathrm{pH} 7$

4-Aminophenazone

Phenol

3,4-Dichlorophenol

Sodium cholate

Fatty alcohol polyethylene glycol ether

Cholesterol esterase

Cholesterol oxidase

$\begin{array}{cl}100 & \mathrm{mmol} / \mathrm{l} \\ 1 & \mathrm{mmol} / \mathrm{l} \\ 6 & \mathrm{mmol} / \mathrm{l} \\ 6 & \mathrm{mmol} / \mathrm{l} \\ 10 & \mathrm{mmol} / \mathrm{l} \\ 3 & \mathrm{~g} / \mathrm{l} \\ 0.4 & \mathrm{kU} / \mathrm{l} \\ 0.25 \mathrm{kU} / \mathrm{l} \\ 0.20 \mathrm{kU} / \mathrm{l}\end{array}$

Peroxidase

Lyophilized sera with cholesterol concentrations measured by isotope dilution mass spectrometry were from NIST (formerly NBS), USA (SRM 909), or were a kind gift from Prof. $L$. Siekmann (Bonn) or from Prof. G. Galli (Milano). Sera with cholesterol concentrations measured by the reference AbellKendall procedure were kindly supplied by Dr. J. C. Koedam (Bilthoven The Netherlands) (two lyophilized preparations), and by Dr. D. G. Bullock (Birmingham) (two liquid specimens). All were of human origin.

The proposed (reference) extraction-enzymatic procedure was performed as previously described (7), with a major modification in the extract-drying step, which will be discussed later.

Briefly, $250 \mu \mathrm{l}$ of serum (or cholesterol solution or ethanol) are mixed with $2250 \mu \mathrm{l}$ of ethanolic potassium hydroxide solution (approximately $9 \mathrm{~mol} / \mathrm{l}$ ), and incubated for 3 hours at $37^{\circ} \mathrm{C}$. Water $(2.5 \mathrm{ml})$ is then added, followed by $5.0 \mathrm{ml}$ of hexane.

After vortexing $(3 \times 20 \mathrm{~s}), 1 \mathrm{ml}$ of the hexane phase is transferred to $16 \times 75 \mathrm{~mm}$ tubes, and dried at $69 \pm 0.5^{\circ} \mathrm{C}$ in a thermostatted aluminium block. Drying is complete in about 45 minutes; tubes are removed from the block as soon as they are dry. Ethanol $(0.5 \mathrm{ml})$ is added to each tube, followed by $3 \times 20 \mathrm{~s}$ vortexing and by the addition of $5.00 \mathrm{ml}$ of freshly reconstituted enzymatic reagent. After 4 minutes incubation at $37^{\circ} \mathrm{C}$, and 4 minutes equilibration at room temperature, absorbance values are measured (blank, calibrators and samples) against water at $495 \mathrm{~nm}$. Readings are taken following the order of reagent addition, but the exact timing is not critical (see later).

For calibration, fresh cholesterol solutions are prepared each time, by weighing 3 different quantities of cholesterol to the nearest $0.01 \mathrm{mg}$, and dissolving them in ethanol with gentle warming. After careful equilibration at $20^{\circ} \mathrm{C}$, volumes are made up to $50.00 \mathrm{ml}$; final concentrations are chosen to cover the range $2.59-10.34 \mathrm{mmol} / 1$. For each analytical series, the three solutions (equilibrated at $20^{\circ} \mathrm{C}$ ), and ethanol for the blank, are pipetted in duplicate together with the samples, and submitted to all the steps of the procedure. The $4 \times 2$ absorbance values are regressed against cholesterol concentration ( 0 in the case of blanks), thereby generating the calibration function used for calculating the concentration in unknowns, and the statistical parameters for the evaluation of the calibration line itself.

In the overall procedure, the critical volumetric steps are:

1) dilution of the $250 \mu \mathrm{l}$ of sample (or ethanol or calibrators) with $2250 \mu \mathrm{l}$ of ethanolic potassium hydroxyde solution: for this, a Microlab $\mathrm{M}$ dilutor (Hamilton) was used;

2) pipetting the $5.00 \mathrm{ml}$ of hexane: for this a Multipette 4780 (Eppendorf) was used;

3) sampling the $1.00 \mathrm{ml}$ of extract: this was done with a positive displacement sampler (macro-Transferpettor, Brand);
4) pipetting the $5.00 \mathrm{ml}$ of enzymatic reagent: this was done with the Hamilton diluter.

Compatibility of the plastic parts of the measuring devices with hexane was tested in advance. Imprecision (CV from 20 replicate measurements) and inaccuracy were checked gravimetrically with the following results: $\leq 0.1 \%$ and $\leq 0.2 \%$ respectively for the diluter; $\leq 0.3 \%$ and $\leq 1.5 \%$ respectively for the other devices.

For direct comparison of the two reactions, aliquots of $1 \mathrm{ml}$ and $2 \mathrm{ml}$ from the same hexane phase were dried in separate tubes, then submitted to the enzymatic reaction as described here $(1 \mathrm{ml}$ aliquot), or to the Liebermann-Burchard reaction ( $2 \mathrm{ml}$ aliquot), by adding $2.50 \mathrm{ml}$ of the reagent (4), incubating 30 minutes at $25^{\circ} \mathrm{C}$ and reading at $630 \mathrm{~nm}$ with accurate timing (4).

Spectrophotometric readings were taken with a Uvicon 860 (Kontron) double beam spectrophotometer with conventional optical-glass cuvettes, light-path $10.0 \mathrm{~nm}$. Alternatively, a mod. CL-750 spectrophotometer (Shimadzu), equipped with flowthrough $10 \mathrm{~mm}$ light-path cell was used, with equivalent results.

All samples were assayed in duplicate (up to 4 replicates in experiments for assessing imprecision). Normally, an analytical series included up to 12 samples, three calibration solutions, one blank and one or two control materials (all assayed in duplicate, total up to 36 tubes). The working time was about 4 hours, excluding hydrolysis and drying time, and the time for the preparations of the calibrators.

\section{Results}

\section{Calibration}

The calibration line was accepted if the following requirements were fulfilled:

$-\mid$ (mean blank absorbance) - (intercept) $\mid \leq 0.0035$ absorbance units;

- difference | (weighed value ) - (calculated value from the equation) $\mid \leq 0.071 \mathrm{mmol} / \mathrm{l}$ for each of the 6 calibrators; standard deviation of the differences $\leq 0.0375 \mathrm{mmol} / \mathrm{l} ; \mathrm{r} \geq 0.9998$.

The long term stability of the calibration, as demonstrated by constancy in the slope value, is shown in table 1. Blank values were in the range $0.022-0.033$ absorbance units.

\section{Drying of the $1 \mathrm{ml}$ aliquot of hexane extract}

As mentioned, we modified our drying technique and therefore we checked for possible lost of cholesterol. Tubes with the same amount of extract were removed from the heating block immediatly after drying or left in the block for additional periods of time, up to 30 minutes. The reaction was then performed on each tube, and the results are shown in figure 1.

\section{Development and stability of the colour}

Dried extracts were submitted to the enzymatic and to the Liebermann-Burchard reactions, and the absorbances of the mixtures, at 495 and $620 \mathrm{~nm}$ respec- 
Tab. 1. Slope of the calibration line, from linear regression analysis of absorbance values (y), in absorbance units, against cholesterol concentration (x), in $\mathrm{mmol} / \mathrm{l}$.

\begin{tabular}{llll}
\hline Time & \multicolumn{3}{l}{ Slope value } \\
\hline March & 1984 & $(68.06 \pm 0)$ & E-03 \\
June & 1984 & $(67.28 \pm 0.39)$ & E-03 \\
June & 1985 & $(66.90 \pm 0)$ & E-03 \\
August & 1985 & $(67.28 \pm 0.39)$ & E-03 \\
August & 1985 & $(68.06 \pm 0.39)$ & E-03 \\
May & 1986 & $(68.44 \pm 0.39)$ & E-03 \\
May & 1986 & $(67.28 \pm 0.39)$ & E-03 \\
May & 1986 & $(68.06 \pm 0.77)$ & E-03 \\
May & 1986 & $(66.90 \pm 0)$ & E-03 \\
June & 1989 & $(66.90 \pm 0.39)$ & E-03 \\
June & 1989 & $(67.28 \pm 0.77)$ & E-03 \\
July & 1989 & $(67.28 \pm 0.39)$ & E-03 \\
July & 1989 & $(68.83 \pm 0)$ & E-03 \\
February & 1990 & $(68.06 \pm 0.39)$ & E-03 \\
March & 1990 & $(68.83 \pm 0.39)$ & E-03 \\
March & 1990 & $(68.44 \pm 0.39)$ & E-03 \\
& & 67.75 & \\
& mean & 0.66 & \\
\hline
\end{tabular}

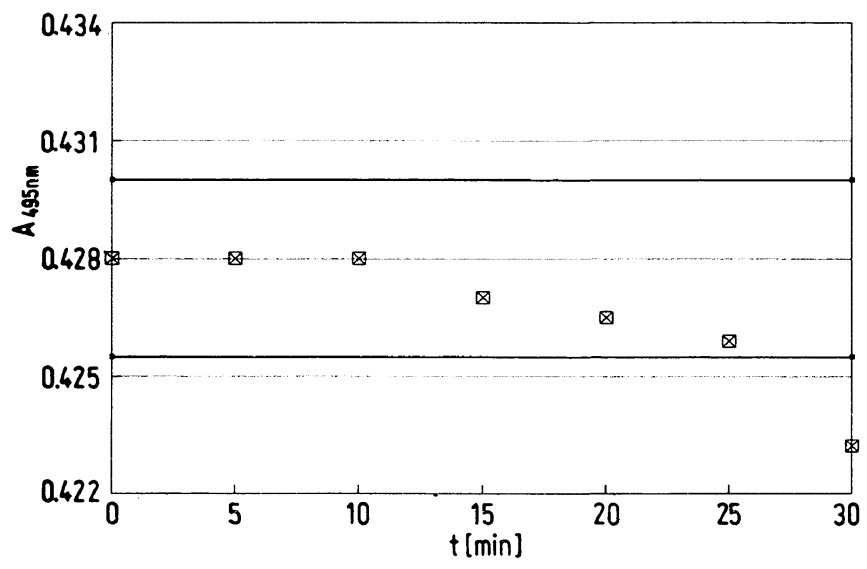

Fig. 1. Effect of leaving the dried extract at $69^{\circ} \mathrm{C}$ (x-axis, minutes) on the recovery of cholesterol (y-axis, absorbance values at $495 \mathrm{~nm}$ following the enzymatic reaction). Continuous lines: mean \pm 2 standard deviations of the absorbance of samples withdrawn from the heating block as soon as dried.

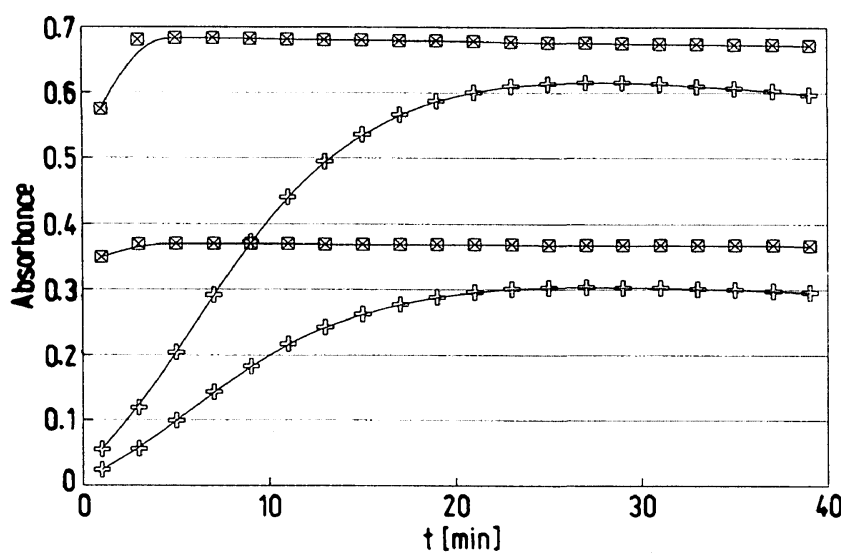

Fig. 2. Time course of the enzymatic (squares) and of the Liebermann-Burchard (crosses) reactions at $25^{\circ} \mathrm{C}$ : two cholesterol levels. $x$-Axis: reaction time (minutes); $y$ axis: absorbance values, at 495 and $620 \mathrm{~nm}$ respectively. tively, were recorded at time intervals at $25^{\circ} \mathrm{C}$. The results are shown in figure 2 . The time-window encompassing absorbance values in the range $99.7-$ $100 \%$ of the maximum value was 10 minutes for the enzymatic reagent, 4 minutes for the LiebermannBurchard reagent. Repeated scanning at 3 minutes intervals, in the range $450-650 \mathrm{~nm}$, showed slow shifting of the absorbance peak of the LiebermannBurchard reaction from 610 to $622 \mathrm{~nm}$ during the course of the reaction, while the absorbance peak of the enzymatic reaction remained constant at $495 \mathrm{~nm}$.

\section{Assessment of imprecision}

In 3 different experiments, two lyophilized sera were assayed, 3 to 4 replicates each, on 4 different days during a period of $19-25$ days. Results were statistically assessed by means of ANOVA, and are shown in table 2. Long-term stability of the method was assessed by assaying two lyophilized sera at time intervals; no significant variation in the measured concentration values was observed over a period of 273 days.

Tab. 2. Evaluation of imprecision. Statistical analysis of results was by means of ANOVA.

\begin{tabular}{|c|c|c|c|c|c|c|}
\hline \multirow{2}{*}{$\begin{array}{l}\text { Exp. } \\
\text { No. }\end{array}$} & \multicolumn{2}{|c|}{ Number of } & \multirow{2}{*}{$\begin{array}{l}\text { Overall } \\
\text { mean } \\
(\mathrm{mmol} / \mathrm{l})\end{array}$} & \multicolumn{3}{|c|}{ Imprecision (CV) } \\
\hline & $\begin{array}{l}\text { repli- } \\
\text { cates }\end{array}$ & $\overline{\text { days }}$ & & $\begin{array}{l}\text { in-the- } \\
\text { series }\end{array}$ & $\begin{array}{l}\text { between- } \\
\text { days }\end{array}$ & overall \\
\hline 1 & 4 & 4 & 4.28 & 0.62 & 0.61 & 0.87 \\
\hline 1 & 4 & 4 & 6.26 & 0.65 & 0.96 & 1.16 \\
\hline 2 & 3 & 4 & 6.17 & 0.59 & 0.49 & 0.77 \\
\hline 2 & 3 & 4 & 7.38 & 0.44 & 0.51 & 0.67 \\
\hline 3 & 3 & 4 & 4.35 & 1.08 & 0 & 1.08 \\
\hline 3 & 3 & 4 & 6.18 & 0.47 & 0.44 & 0.64 \\
\hline
\end{tabular}

\section{Assessment of inaccuracy}

Comparison with the definitive method was achieved by measuring cholesterol concentration in lyophilized materials from different sources, which had been assayed in other laboratories by means of isotope delution mass spectrometry; the results are shown in table 3. Comparison with the reference Abell-Kendall procedure was performed in a similar way (results in tab. 4). The enzymatic and the Liebermann-Burchard reactions were also compared directly by measuring the cholesterol concentrations of 50 fresh serum samples, selected to continuously cover the range 3.43$8.41 \mathrm{mmol} / \mathrm{l}$; both reactions were applied to dried aliquots of the same extracts; the results are shown in figure 3. Linear regression-correlation analysis by an ordinary least-square model gave a slope value not different from $1(1.001 \pm 0.007)$ and an intercept $(-0.052 \mathrm{mmol} / \mathrm{l})$ significantly different from 0 ; the residual standard deviation $\left(\mathrm{S}_{\mathrm{yx}}\right)$ was $0.08 \mathrm{mmol} / \mathrm{l}$. 
Tab. 3. Evaluation of inaccuracy, by measurement of cholesterol concentration in lyophilized materials previously assayed by definitive method (isotope dilution mass spectrometry, IDMS).

\begin{tabular}{|c|c|c|c|c|c|}
\hline \multicolumn{2}{|c|}{ Material } & \multirow{2}{*}{$\begin{array}{c}\text { Year } \\
1984\end{array}$} & \multirow{2}{*}{$\begin{array}{l}\begin{array}{l}\text { IDMS- } \\
\text { value }\end{array} \\
(\mathrm{mmol} / \mathrm{l}) \\
3.69\end{array}$} & \multirow{2}{*}{ 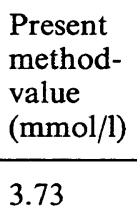 } & \multirow{2}{*}{$\begin{array}{l}\begin{array}{l}\text { Percent } \\
\text { differ- } \\
\text { ence* }\end{array} \\
+1.1\end{array}$} \\
\hline SRM & 909 \# & & & & \\
\hline 319 & $* *$ & 1984 & 2.88 & 2.97 & +3.1 \\
\hline 378 & $* *$ & 1984 & 2.60 & 2.60 & 0 \\
\hline 380 & $* *$ & 1984 & 2.43 & 2.43 & 0 \\
\hline 381 & $* *$ & 1984 & 2.83 & 2.82 & -0.4 \\
\hline A & \# \# & 1989 & 4.46 & 4.30 & -3.6 \\
\hline B & \# \# & 1989 & 6.16 & 6.26 & +1.6 \\
\hline A & \# \# & 1990 & 4.46 & 4.35 & -2.5 \\
\hline \multirow[t]{2}{*}{ B } & \# \# & 1990 & 6.16 & 6.18 & +0.3 \\
\hline & & & & mean & -0.4 \\
\hline
\end{tabular}

* [(present method-value)-(IDMS-value) $] \times 100$

\# obtained from NIST (formerly NBS)

** $\quad$ kindly supplied by Prof. L. Siekmann (Bonn)

\# \# IDMS-assay performed by Prof. G. Galli (Milano)

Tab. 4. Evaluation of inaccuracy, by measurement of cholesterol concentration in materials previously assayed with the reference (Abell-Kendall, AK) method.

\begin{tabular}{|c|c|c|c|c|}
\hline Material & Year & $\begin{array}{l}\text { AK-value } \\
(\mathrm{mmol} / \mathrm{l})\end{array}$ & $\begin{array}{l}\text { Present } \\
\text { method- } \\
\text { value } \\
(\mathrm{mmol} / \mathrm{l})\end{array}$ & $\begin{array}{l}\text { Percent } \\
\text { difference* }\end{array}$ \\
\hline A \# & 1984 & 3.96 & 3.96 & 0 \\
\hline B \# & 1984 & 8.18 & 8.15 & -0.4 \\
\hline $\mathrm{A} 1 * *$ & 1990 & 7.37 \# \# & 7.38 & +0.1 \\
\hline \multirow[t]{2}{*}{$\mathrm{B} 1 * *$} & 1990 & $6.20 \# \#$ & 6.17 & -0.2 \\
\hline & & & mean & -0.1 \\
\hline
\end{tabular}

* [(present method-value $)-($ AK-value $)] \times 100$

\# kindly supplied by Dr. J. C. Koedam (Bilthoven)

** kindly supplied by Dr. D. G. Bullock (Birmingham)

\# \# mean value from two independent laboratories

\section{Discussion}

The described procedure is substantially a scaleddown AK-CDC procedure (4), including somewhat more drastic hydrolysis conditions (3) and substitution of the enzymatic reagent for the LiebermannBurchard reagent. Compared with the LiebermannBurchard reagent, the enzymatic reagent is faster to prepare, and easier and safer to handle. If this advantage is considered, together with the higher stability of the final colour, and the lack of necessity for accurately timed readings, it may be concluded that the goal of increasing the practicability of the reference procedure has been achieved. The reagent may be prepared in the laboratory, but we it found easier and safer to use a prepacked reagent; indeed, over a

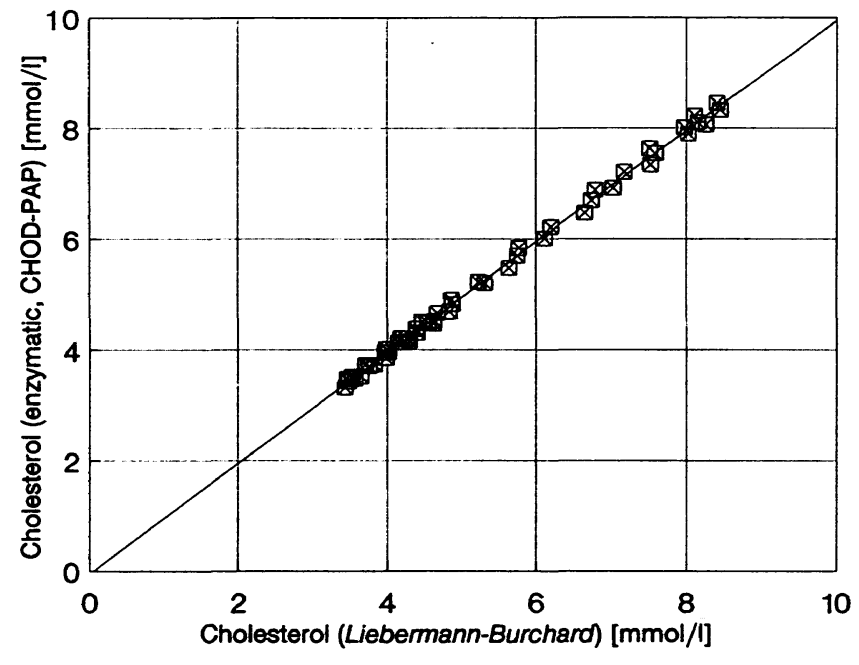

Fig. 3. Cholesterol concentration in 50 patient sera, measured by the Liebermann-Burchard reaction (x-axis, mmol/l) and the enzymatic cholesterol oxidase/4-aminophenazone (CHOD-PAP) reaction (y-axis, $\mathrm{mmol} / \mathrm{l}$ ), following a common hydrolysis/extraction step. Results of statistical analysis are given in the text.

period of 6 years, using several different batches of the commercially available reagent, we obtained very reproducible calibration lines (tab. 1), as well as constant blank values. The cholesterol esterase in the mixture is redundant, but it does not interfere.

In developing the method, the main problem was dissolving the dried extract, which does not directly dissolve in the enzymatic reagent as it does in the Liebermann-Burchard reagent. Therefore we tested the compatibility of several alcohols with the reagent itself (10). The best results were obtained with ethanol, which was found not to interfere with the reaction (kinetics and linearity) up to a volume fraction of 0.17 . With the volume fraction used here $(0.09)$, we recorded a $7.9 \%$ increase in the slope of the calibration line, and a small shift (from 498 to $495 \mathrm{~nm}$ ) of the absorbance peak; this has no effect on the accuracy, since the same ethanol concentration is present in blank, calibrators and samples. With different reagent formulations, problems may possibly arise from interaction of the alcohol with the detergent.

Although the "direct" enzymatic method may give results acceptably well correlated with a reference procedure (11), its use as a reference method has been reported to be unsuitable (12). The direct approach may give inaccurate values because of:

a) incomplete hydrolysis of the esters $(13-15)$;

b) occurrence of turbidity;

c) competitive consumption of hydrogen peroxide by the matrix. 
By means of appropriate experimental design we measured the effect of events b) and c) and found them to be widely variable from serum to serum. Following the hydrolysis/extraction step, such events are unlikely to occur. The conditions adopted here have been chosen to allow for substantially complete hydrolysis of cholesteryl esters (3). We found also that concentrations of bilirubin and ascorbic acid, giving respectively $16 \%$ and $96 \%$ inhibition of the direct reaction, had no measurable effect after the hydrolysis/extraction step (7). Furthermore, spectrophotometric scanning of the final reaction mixture from several serum samples, in comparison with calibrators, failed to reveal any evidence of turbidity.

The overall analytical imprecision (CV) was consistently $<1.5 \%(16)$, rarely exceeding $1 \%$. Major sources of imprecision were found to be the critical volumetric steps, particularly the sampling of the $1 \mathrm{ml}$-aliquot of the hexane phase, which is also part of the accepted reference method. Slight inaccuracy in the volume-measuring devices is without effect on the overall precision, if the same device is used throughout the whole analytical series. The long term stability of the procedure was also found to be satisfactory.

The accuracy of the method, as determined by comparing isotope dilution mass spectrometry-values and Abell-Kendall-reference-values obtained in other laboratories, was satisfactory, although up to +3.1 and $-3.6 \%$ differences were found in two instances. Direct comparison of the enzymatic and the LiebermannBurchard reactions on 50 fresh serum samples, in an experiment designed to minimize sources of discrepancies not due to the reactions, gave a constant bias (enzymatic vs Liebermann-Burchard) of -0.052 $\mathrm{mmol} / \mathrm{l}$, corresponding to about $0.8 \%$ at the $6 \mathrm{mmol} / \mathrm{l}$ mean concentration value. It is tempting to speculate that sera contain a constant amount of non-cholesterol extractable (not identified) material, able to interfere in the Liebermann-Burchard reaction but not in the (theoretically) more specific enzymatic reaction. As a matter of fact, the AK-CDC procedure has been found to have a $1.5 \%$ systematic proportional bias with respect to the isotope dilution mass spectrometry procedure, and this has been attributed to the presence of unidentified interfering components (11). The low specificity of the Liebermann Burchard reaction, with its undefined final product(s), is well documented (4).

It can be concluded that the herein proposed procedure is more practicable than the AK-CDC method, whilst exhibiting comparable precision and, possibly, better accuracy. More extensive comparison with the mass spectrometry-based procedures, which allow high-accuracy measurement of serum cholesterol concentration $(5,17,18)$, might better substantiate this last point. Currently transferability studies (16) should demonstrate the robustness of the method. The results reported were obtained in two laboratories $(6,7)$ over a 6-years period, without any evidence of discrepancies in results.

A detailed protocol of the procedure is available upon request.

\section{Acknowledgement}

Prof. L. Siekmann (Bonn), Prof. G. Galli (Milano), Dr. J. C. Koedam (Bilthoven) and Dr. D. G. Bullock (Birmingham) kindly supplied materials assayed by means of isotope dilution mass spectrometry and Abell-Kendall reference methods. Cooperation with Prof. G. Galli (Milano) was part of the (Italian) National Programme for Education in Cholesterol Control (Coordinator: Prof. R. Paoletti, Milano). The secretarial assistance of Miss I. Moraschinelli is gratefully acknowledged.

\section{References}

1. Tietz, N. W. (1979) A model for comprehensive measurement system in clinical chemistry. Clin. Chem. 25, 833839.

2. Cooper, G. R. \& Myers, G. L. (1990) Reference system for cholesterol measurements. Scand. J. Clin. Lab. Invest. 50, Suppl. 198, 27-31.

3. Cohen, A., Hertz, H. S., Mandel, J., Paule, R. C., Schaffer, R., Sniegoski, L. T., Sun, T., Welch, M. J. \& White, V. E. (1980) Total serum cholesterol by isotope dilution/mass spectrometry: a candidate definitive method. Clin. Chem. $26,854-860$.

4. Duncan, I. W., Mather, A. \& Cooper, G. R. (1982) The procedure for the proposed cholesterol reference method. Clinical Chemistry Division. Centers for Disease Control, Atlanta, GA, 75 pp.

5. Derks, H. J. G. M., van Heiningen, A. \& Koedam, H. C. (1985) Gas-chromatographic determination of cholesterol in serum: candidate reference method. Clin. Chem. 31, $691-694$.

6. Franzini, C. \& Besozzi, M. (1984) Proposed "candidate reference method" for the determination of total serum cholesterol (Abst.). XII Int. Cong. Clin. Chem. Rio de Janeiro, Brazil. Abstracts Volume, 104.

7. Franzini, C. \& Besozzi, M. (1985) Proposed reference method for serum cholesterol assay. Giorn. It. Chim. Clin. $10,251-256$.

8. Allain, C. C., Poon, L. S., Chan, C. S. G., Richmond, W. \& Fu, P. C. (1974) Enzymatic determination of total serum cholesterol. Clin. Chem. 20, 470-475.

9. Kattermann, R., Jaworek, D. \& Moeller, G. (1984) Multicentre study of a new enzymatic method of cholesterol determination. J. Clin. Chem. Clin. Biochem. 22, 245-251.

10. Franzini, C. (1985) Alcoholic versus aqueous calibrators for the enzymatic assay of serum cholesterol. J. Clin. Chem. Clin. Biochem. 23, 105

11. Bachorik, P. S., Virgil, D. G., Derby, C., Vidman, D., Mc Mahon, R., Eulwood, R. T. \& Ezzati, T. (1988) Enzymatic analysis of total- and HDL-cholesterol: comparison with

J. Clin. Chem. Clin. Biochem. / Vol. 28, 1990 / No. 12 
the standardized Liebermann-Burchard method used by the lipid research clinics programme. Clin. Chim. Acta. 174, $307-314$.

12. Ellerbe, P., Myers, G. L., Cooper, G. R., Hertz, H. S., Sniegoski, L. T., Welch, M. J. \& White, V. E. (1990) A comparison of results for cholesterol in human serum obtained by the reference method and by the definitive method of the national reference system for cholesterol. Clin. Chem. $36,370-375$.

13. Tel, R. M. \& Berends, G. T. (1980) Incomplete hydrolysis of cholesteryl esters during the enzymatic cholesterol determination as evidenced by aqueous cholesteryl ester solutions: comparison of six enzymatric procedures with the Liebermann-Burchard method. J. Clin. Chem. Clin. Biochem. 18, 595-601.

14. Buongiorno, A. M., Macchia, T., Mancinelli, R. \& Morisi, G. (1983) Caratterizzazione di alcuni metodi enzimatici automatizzati per la determinazione del colesterolo sierico totale. Studio dell' efficacia dell'enzima colesteroloesterasi nella reazione d'idrolisi degli esteri del colesterolo. Giorn. It. Chim. Clin. 8, 219-229.
15. Wiebe, D. A. \& Bernert, Jr., J. T. (1984) Influence of incomplete cholesteryl ester hydrolysis on enzymic measurements of cholesterol. Clin. Chem. 30, 352-356.

16. Cooper, G. R., Smith, S. J., Duncan, I. W., Mather, A.. Fellows, W. D., Foley, T., Frantz, Jr., I. D., Gill, J. B., Grooms, T. A., Hynie, I., Leassig, R., LoBasso, F. A.. Martin, J., Naito, H., Newman, H. A., Sideman, L., Turner, J. H. \& Williams, D. (1986) Interlaboratory testing of the transferability of a candidate reference method for total cholesterol in serum. Clin. Chem. 32, 921-929.

17. Siekmann, L., Huskes, K. P. \& Breuer, H. (1976) Determination of cholesterol in serum using mass fragmentography - a reference method on Clinical Chemistry. Fresenius J. Anal. Chem. 279, 145-146.

18. Freudenthal, J., Derks, H. J. G. M., Gramberg, C. G., tenHove, G. J. \& Klassen, R. (1981) Isotope dilution mass spectrometry of cholesterol in serum. Biomed. Mass Spectrom. 8, 5-9.

Prof. Carlo Franzini

Ospedale di Rho

Laboratorio Analisi

Corso Europa, 250

I-20017 RHO (Milano) 